\title{
Guest editorial: web applications and techniques
}

\author{
Wei Wang ${ }^{1} \cdot$ Wenjie Zhang ${ }^{1}$
}

Published online: 24 May 2015

(C) Springer Science+Business Media New York 2015

Intense and continuous development of Web functionalities and technologies has empowered nearly all aspects of daily life, and changed the way people and organizations communicate and interact. This special issue collects five research papers which are extensions of the best papers of the APWeb 2013 conference (The 15th Asia-Pacific Web Conference, Sydney, Australia). These papers study interesting and novel problems in Web technologies, database systems, and information management.

The first paper, by Unankard, Li, and Sharaf, "Emerging Event Detection in Social Networks with Location Sensitivity", focuses on event detection in social networks. It proposes an approach for early detection of merging hotspot events in social networks with local sensitivity. Strong correlations are identified between user locations and event locations to detect emerging events.

The second paper, by Zhang et al., "Detecting Anomaly in Data Streams by Fractal Model", addresses the limitations of existing work in detecting anomaly in data streams. The authors propose a fractal model-based approach with a history based and a parameter-free algorithm which consumes only limited amount of memory and does not involve any training process.

Kuwada et al. study the consistency of XML schema mapping in the third paper, "The Consistency and Absolute Consistency Problems of XML Schema Mappings between Restricted DTDs". The paper discusses the consistency and absolute consistency problems under restricted DTDs called disjunction-capsuled DTDs, and shows that both problems are solvable in polynomial time under disjunction-capsuled DTDs.

In the fourth paper, "Multi-verifier: A Novel Method for Fact Statement Verification", Wang, Zhu, and Wang study the problem of fact statement verification. A method named multi-verifier is proposed to determine the truthfulness of a fact statement. In this method, a popular search engine is utilized to collect the top-n search results that are most related to the target fact statement. The authors also proposed the concepts of support score and credibility ranking for verification.

Wenjie Zhang

zhangw@cse.unsw.edu.au

Wei Wang

weiw@cse.unsw.edu.au

1 The University of New South Wales, Sydney, Australia 
Finally, the paper "Privacy Preserving Graph Publication in a Distributed Environment", by Yuan et al., focuses on privacy preserving data publishing in social networks, where each publisher only holds a subgraph of the complete graph. A SMC (Secure Multi-Party Computation) based protocol is proposed to publish a privacy preserving graph in a distributed environment. This protocol publishes a privacy preserving graph without leaking the local content information and achieving the maximum graph utility.

We thank the authors for their contributions in this special issue. We also thank the reviewers for their valuable comments throughout the rigours review process. We hope that these papers provide the community with insights and inspirations for continued research work. 